\title{
The stability and gravitational Newtonian limit of a modified Randall-Sundrum model
}

\author{
Shahrokh Parvizi ${ }^{\mathrm{a}}$, Mojtaba Shahbazi ${ }^{\mathrm{b}}$ \\ Department of Physics, School of Sciences, Tarbiat Modares University, P.O. Box 14155-4838, Tehran, Iran
}

Received: 14 October 2015 / Accepted: 29 December 2015 / Published online: 19 January 2016

(c) The Author(s) 2016. This article is published with open access at Springerlink.com

\begin{abstract}
For a modified Randall-Sundrum model (Jones et al. in Phys. Rev. D 88:025048, 2013), the graviton equations are derived and the mass spectrum found. The latter includes a massless graviton and a continuum mass with a gap. There is no negative mass-squared in the spectrum, so the model is stable. The gravitational Newtonian limit is obtained with an exponentially suppressed modification from the extra dimension.
\end{abstract}

\section{Introduction}

Extra dimensional brane-world models were first introduced in [1] and then in [2] (see also [3]) to solve the hierarchy problem in fundamental interactions including gauge fields and gravity. The basic idea of these models and their followers is an assumption that matter and gauge fields are confined on a three-dimensional brane embedded in a higher dimensional spacetime, while the gravity, by definition, can travel in all dimensions. This is compatible with observations, provided either the extra dimension volume is of the order of the $\mathrm{TeV}$ scale as suggested in [1] or somehow warped over the brane as in [2].

The mentioned assumption is supported by string theory in which (D-)branes are defined to be where open strings ended. The latter correspond to the standard model fields in the low energy limit. In contrast, closed strings can propagate into extra dimension(s) off the brane and in the low energy limit correspond to gravitons. Despite this justification, it is important to understand the mechanism of field localization on the brane in the low energy scales and independent of the string theory. This was not very satisfactory and all Standard Model's particles could not be trapped into the brane in the original five-dimensional version of this model, though there are successes by using some six-dimensional models [4-7].

\footnotetext{
a e-mail: parvizi@modares.ac.ir

b e-mail: Mojtaba.Shahbazi@modares.ac.ir
}

In this regard, in [8], a five-dimensional modified RandallSundrum (MRS) model was proposed and shown that it improves the field localization behavior on the brane. This model, locally, looks very similar to the original RS, but it was shown that they differ globally [8]. An important difference is that, in contrast to the RS model, the new one does not contain any five-dimensional cosmological constant.

Regarding progress in the field localization by proposing [8], it is worth examining the effective four-dimensional gravitational behavior of this model. In this article, we investigate the gravitational perturbation of the background and find the graviton spectrum. We show that it excludes negative mass modes which signing stability of the model. Moreover, its Newtonian gravity limit is studied.

The organization of this paper is as follows. In Sect. 2 we introduce the metric and recall its differences with the RS. In Sect. 3, we perturb the background and find the graviton mass spectrum. In Sect. 4, we study the Newtonian limit and conclude in Sect. 5.

\section{The background metric}

Consider the five-dimensional Einstein-Hilbert gravity action, which reads as follows:

$S_{g}=\frac{1}{2 \kappa^{2}} \int \mathrm{d}^{5} x \sqrt{\mid g^{(5)}} \mid R+S_{\text {brane }}+S_{\text {matter }}$

in which $S_{\text {brane }}$ is a localized action of brane(s) and $S_{\text {matter }}$ is the action for any matter in the system. In the next section it will be shown that $\mu \nu$-component of energy-momentum tensor comprises two terms, a delta Dirac term which indicates the brane and a constant term which could emanate from a scalar source field [8].

Based on this action, the following metric can be introduced as a solution to the equations of motion which contains a 
3-brane at the origin of fifth dimension [8]:

$\mathrm{d} s^{2}=e^{-2 k|r|} \eta_{A B} \mathrm{~d} x^{A} d x^{B}=e^{-2 k|r|}\left(\eta_{\mu \nu} \mathrm{d} x^{\mu} \mathrm{d} x^{\nu}+\mathrm{d} r^{2}\right)$

where $r$ is the extra dimension varying from $-\infty$ to $+\infty$ and the Latin letters are from 1 to 5 and Greek letters (brane coordinates) from 1 to 4 . In addition, $\eta_{\mu \nu}$ is Minkowskian metric with $\eta_{\mu \nu}=\operatorname{diag}(-1,1,1,1)$. Henceforth, we use the nomination of [8] and call (2) the $r$-metric.

It is worth recalling the well-known Randall-Sundrum (RS) metric [9]:

$\mathrm{d} s_{R S}^{2}=e^{-2 k|z|} \eta_{\mu \nu} \mathrm{d} X^{\mu} \mathrm{d} X^{\nu}+\mathrm{d} z^{2}$.

At first sight it seems that the $r$-metric (2) can be converted to the RS metric (3), by the following transformation [8]:

$$
\begin{aligned}
& e^{-k|r|}=1-k|z| \\
& \mathrm{d} x^{\mu}=\frac{e^{-k|z|}}{1-k|z|} \mathrm{d} X^{\mu} .
\end{aligned}
$$

However, these two metrics cannot be for the same spacetime for at least two reasons [8]: First, the above coordinate transformation is singular at $z=1 / k$, so it cannot be extended to all space. Second, a global coordinate transformation needs to be an exact differential as $\mathrm{d} x^{\mu}=\partial x^{\mu} / \partial X^{\nu} \mathrm{d} X^{\nu}+\partial x^{\mu} / \partial z \mathrm{~d} z$, which is manifestly not the case. Moreover, it is shown in [8] that the consistent Einstein equation including the energymomentum of the brane requires the cosmological constant to be zero as we already have set in (1), in contrast to the Randall-Sundrum model, which contains a negative fivedimensional cosmological constant. Furthermore the field localization of different spin fields gets an improvement compared to the original Randall-Sundrum model [8].

\section{Field equations and Graviton modes}

To derive Einstein's field equations, for the matter of convenience, a different coordinate system is adopted:

$\mathrm{d} s^{2}=e^{2 \sigma}\left(\eta_{\mu \nu} \mathrm{d} x^{\mu} \mathrm{d} x^{\nu}\right)+\mathrm{d} y^{2}$

with $\sigma=\ln (1-k|y|)$. By inserting (5) in the Einstein's equation:

$\kappa^{2} T_{\mu \nu}=g_{\mu \nu}\left(3 \ddot{\sigma}+6 \dot{\sigma}^{2}\right)$,

$\kappa^{2} T_{55}=6 \dot{\sigma}^{2}$,

where a dot represents the derivative with respect to the $y$ extra dimension.

To find graviton modes in the MRS model, the metric would be perturbed around the fixed background (5) as fol- lows:

$G_{A B}=g_{A B}+h_{A B}$.

Plug (8) into (1) and keep up to second order in $h$, then the variation due to $h^{A B}$ gives the linearized equation of motion [9]:

$$
\begin{aligned}
& \nabla^{T} \nabla_{N} h_{M T}+\nabla^{T} \nabla_{T} h_{M N}-\nabla_{M} \nabla_{N} h-g_{M N} \nabla^{T} \nabla^{S} h_{T S} \\
& +\nabla^{T} \nabla_{T} h-2 \kappa^{2}\left(T_{M T} h_{N}^{T}+T_{N T} h_{M}^{T}-\frac{1}{2} T_{M N} h\right. \\
& \left.\quad-\frac{1}{2} g_{M N} T_{T S} h^{T S}-\frac{T_{S}^{S}}{6}\left(2 h_{M N}-g_{M N} h\right)\right) \\
& +\kappa^{2} \mathscr{L} h_{M N}=0
\end{aligned}
$$

where $h=h_{A}^{A}$ and $T_{M N}$ is from (6) and (7). It could be shown that adopting the unitary gauge is feasible [10]:

$h_{\mu 5}=0 \quad$ and $\quad h_{55}=f\left(x^{\mu}\right) e^{n \sigma}:=\varphi$

where $n$ is a constant. By rewriting the equation of motion (9) for different components we get

$\mu \nu$-component:

$$
\begin{aligned}
& \frac{1}{2}\left(\partial_{\rho} \partial^{\rho} h_{\mu \nu}-\partial_{\mu} \partial^{\rho} h_{\rho \nu}-\partial_{\nu} \partial^{\rho} h_{\rho \mu}+\ddot{h}_{\mu \nu}\right)+4 \dot{\sigma}^{2} h_{\mu \nu} \\
& \quad+\frac{1}{2} \partial_{\mu} \partial_{\nu} \tilde{h}+\frac{1}{2} \partial_{\mu} \partial_{\nu} \varphi+\frac{1}{2} g_{\mu \nu}\left[\partial^{\rho} \partial^{\sigma} h_{\rho \sigma}-\partial_{\rho} \partial^{\rho} \tilde{h}-\ddot{\tilde{h}}\right. \\
& \left.\quad-4 \dot{\sigma} \dot{\tilde{h}}-\partial_{\mu} \partial_{\nu} \varphi-6 \dot{\sigma}^{2} \tilde{h}+3 \dot{\sigma}^{2} \varphi(2+n)\right] \\
& \quad+\ddot{\sigma}\left(\frac{3}{2} g_{\mu \nu} \varphi+2 h_{\mu \nu}-\frac{3}{2} g_{\mu \nu} \tilde{h}\right)-\left(3 \ddot{\sigma}+6 \dot{\sigma}^{2}\right) h_{\mu \nu}=0
\end{aligned}
$$

$\mu 5$-component:

$\frac{1}{2} \partial_{5}\left(\partial_{\mu} \tilde{h}-\partial^{v} h_{\mu \nu}\right)-\frac{3}{2} \dot{\sigma} \partial_{\mu} \varphi=0$,

55-component:

$$
\begin{aligned}
& \frac{1}{2}\left(\partial^{\mu} \partial^{\nu} h_{\mu \nu}-\partial_{\mu} \partial^{\mu} \tilde{h}\right)-\frac{3}{2} \dot{\sigma} \dot{\tilde{h}} \\
& \quad+(13+2 n) \dot{\sigma}^{2} \varphi-3 \dot{\sigma}^{2} \tilde{h}=0,
\end{aligned}
$$

in which $\tilde{h}=g^{\mu \nu} h_{\mu \nu}$. Equations (11-13) are a system of coupled partial differential equations which can be solved if one decouples them. To do this, we use the following tensor decomposition [11]:

$h_{\mu \nu}=E g_{\mu \nu}+B_{, \mu \nu}+C_{\mu, \nu}+C_{\nu, \mu}+D_{\mu \nu}$

where $E$ and $B$ are scalars (called radion in the context of the RS model), $C_{\mu}$ is a divergenceless vector and $D_{\mu \nu}$ a 
traceless-divergenceless tensor (graviton). Then inserting this in the equations of motion one finds

$\mu \nu$-component:

$$
\begin{aligned}
& \frac{1}{2}\left(-2 E_{, \rho}^{\rho} g_{\mu \nu}-C_{\mu, \nu \rho}^{\rho}-C_{\nu, \mu \rho}^{\rho}+D_{\mu \nu, \rho}^{\rho}+2 E_{, \mu \nu}\right. \\
& \left.\quad+\left(E g_{\mu \nu}\right)_{, 55}+B_{, \mu \nu 55}+C_{\mu, \nu 55}+C_{v, \mu 55}+D_{\mu \nu, 55}\right) \\
& \quad+\frac{1}{2} \varphi_{, \mu \nu}+4 \dot{\sigma}^{2}\left(-2 E g_{\mu \nu}+B_{, \mu \nu}+C_{\mu, \nu}+C_{\nu, \mu}+D_{\mu \nu}\right) \\
& \quad+\frac{1}{2} g_{\mu \nu}\left(-4 \ddot{E}-B_{, \rho 55}^{\rho}-4 \dot{\sigma}\left(4 \dot{E}+B_{, \rho 5}^{\rho}\right)-\varphi_{, \rho}^{\rho}-6 B_{, \rho}^{\rho} \dot{\sigma}^{2}\right. \\
& \left.\quad+3 \dot{\sigma}^{2} \varphi(2+n)\right)+\ddot{\sigma}\left(\frac{3}{2} g_{\mu \nu} \varphi-4 g_{\mu \nu} E+2 B_{, \mu \nu}+2 C_{\mu, \nu}\right. \\
& \left.\quad+2 C_{\nu, \mu}+2 D_{\mu \nu}-\frac{3}{2} g_{\mu \nu} B_{, \rho}^{\rho}\right)=0
\end{aligned}
$$

$\mu 5$-component:

$\frac{3}{2} \dot{E}_{, \mu}-\frac{3}{2} \dot{\sigma} e^{n \sigma} f_{, \mu}-\frac{1}{2} \dot{C}_{\mu, \nu}^{\nu}=0$,

55-component:

$$
\begin{aligned}
& -\frac{3}{2} \dot{\sigma} g^{\mu \nu} \dot{B}_{, \mu \nu}+(13+2 n) \dot{\sigma}^{2} e^{n \sigma} f-\frac{3}{2} E_{, \mu}^{\mu}-12 \dot{\sigma}^{2} E-6 \dot{\sigma} \dot{E} \\
& \quad=0 .
\end{aligned}
$$

One of the advantages of the decomposition (14) is that it enables one to make a distinction between the degrees of freedom of the model. Since we are interested in studying the effects of the graviton, we can consistently set equal to zero all parts of the decomposition (14) but the tensorial part $D_{\mu \nu}$. Having done so, the only non-trivial equation would be the $\mu \nu$-component of the equation of motion:

$\partial_{\rho} \partial^{\rho} D_{\mu \nu}+\ddot{D}_{\mu \nu}-\left(4 \dot{\sigma}^{2}+2 \ddot{\sigma}\right) D_{\mu \nu}=0$.

Let us solve (18) by two boundary conditions. The first one is

$\lim _{y \rightarrow \frac{1}{k}} D_{\mu \nu}(x, y)=0$

and the second one is canceling out coefficients of the Dirac delta in (18).

By imposing the Fourier transform along the brane coordinates on Eq. (18):

$e^{-2 \sigma} p^{2} \tilde{D}_{\mu \nu}-\ddot{\tilde{D}}_{\mu \nu}+\left(4 \dot{\sigma}^{2}+2 \ddot{\sigma}\right) \tilde{D}_{\mu \nu}=0$

where $p^{2}:=\eta^{\mu v} p_{\mu} p_{v}=-m^{2}$. Equation (20) is called a Schrodinger-like equation and can be solved to find

$$
\tilde{D}_{\mu \nu}=w_{\mu \nu} e^{n_{1} \sigma}+u_{\mu \nu} e^{n_{2} \sigma}
$$

where $w_{\mu \nu}$ and $u_{\mu \nu}$ are some constant tensors, and

$n_{1}=\frac{1}{2}+\sqrt{\frac{9}{4}-\frac{m^{2}}{k^{2}}}, \quad n_{2}=\frac{1}{2}-\sqrt{\frac{9}{4}-\frac{m^{2}}{k^{2}}}$.

Considering the second boundary condition results in

$\left(w_{\mu \nu}\left(2-n_{1}\right)+u_{\mu \nu}\left(2-n_{2}\right)\right) \delta(y)=0$

$w_{\mu \nu}=-\frac{3+a}{3-a} u_{\mu \nu}:=F u_{\mu \nu}$,

where

$F=-\frac{3+a}{3-a}, \quad a=\sqrt{9-\frac{4 m^{2}}{k^{2}}}$.

Two boundary conditions in (19) and (23) make four cases:

1. $m^{2}=0$ then $n_{1}=2, n_{2}=-1$ and by condition (23), $u_{\mu \nu}=0$. So $w_{\mu \nu}$ can be found by normalizing the solution as follows:

$$
\begin{aligned}
& \int_{-1 / k}^{1 / k} e^{-\sigma} D_{\mu \nu}^{2} \mathrm{~d} y=1, \\
& \left|w_{\mu \nu}\right|^{2} \int e^{\left(2 n_{1}-1\right) \sigma} \mathrm{d} y=\left|w_{\mu \nu}\right|^{2} \int(1-k|y|)^{3} \mathrm{~d} y=1, \\
& \left|w_{\mu \nu}\right|^{2}=2 k .
\end{aligned}
$$

For later use we find

$$
\left.D_{\mu \nu}^{2}\right|_{y=0}=2 k
$$

2. Either $m^{2}<0$ or $0<m^{2} \leq 2 k^{2}$, then the solution (21) does not meet the boundary conditions, since by (19), $n_{2}$ is negative, and we should take $u_{\mu \nu}=0$. However, (23) then implies $w_{\mu \nu}=u_{\mu \nu}=0$. So there is no graviton. Indeed by (23), as long as $n_{1}, n_{2} \neq 2, w_{\mu \nu}$, and $u_{\mu \nu}$ are either both zero or non-zero, thus $n_{1}$ and $n_{2}$ should be both non-negative due to the first boundary condition. The massless graviton is an exception, since $n_{1}=2$ for $m^{2}=0$.

3. $2 k^{2}<m^{2} \leq \frac{9}{4} k^{2}$, and by using (23), $w_{\mu \nu}$ is proportional to $u_{\mu \nu}$, so to find them, we normalize the solution to 1 :

$$
\begin{aligned}
& \int_{-\frac{1}{k}}^{\frac{1}{k}} e^{-\sigma} D_{\mu \nu}^{2} \mathrm{~d} y=1, \\
& \left|u_{\mu \nu}\right|^{2} \int\left(F^{2}(1-k|y|)^{a}+(1-k|y|)^{-a}-2 F\right) \mathrm{d} y=1, \\
& \left|u_{\mu \nu}\right|^{2}=\frac{k}{2\left(\frac{F^{2}}{1+a}+\frac{1}{1-a}-2 F\right)} .
\end{aligned}
$$


Then

$\left.D_{\mu \nu}^{2}\right|_{y=0}=\left|u_{\mu \nu}\right|^{2}(F-1)^{2}=k \frac{m^{2}-2 k^{2}}{m^{2}-k^{2}}$.

4. $\frac{9}{4} k^{2}<m^{2}$ then $n_{1,2}=(1 \pm i|a|) / 2$, using (23) and normalizing the solution to 1 , we find

$$
\begin{aligned}
& \int_{-\frac{1}{k}}^{\frac{1}{k}} e^{-\sigma} D_{\mu \nu}^{2} \mathrm{~d} y=1 \\
& \left|u_{\mu \nu}\right|^{2} \int\left(|F|^{2}+1-F(1-k|y|)^{+i a}\right. \\
& \left.-F^{*}(1-k|y|)^{-i a}\right) \mathrm{d} y=1 \\
& \left|u_{\mu \nu}\right|^{2}=\frac{k}{2\left(|F|^{2}+1-2 \operatorname{Re}\left(\frac{F}{1+i a}\right)\right)}
\end{aligned}
$$

Then

$$
\left.D_{\mu \nu}^{2}\right|_{y=0}=\left|u_{\mu \nu}\right|^{2}\left(|F|^{2}+1-2 \operatorname{Re}(F)\right)=k \frac{m^{2}-2 k^{2}}{m^{2}-k^{2}} .
$$

Note that the final results in Eqs. (26) and (27) are the same.

Before going on it is worth commenting on these results. First, there is no negative mass-squared graviton. This implies the stability of the background metric against tensorial perturbations. Our analysis reveals that there is a massless mode which as we will see in the next section is important to reach a Newtonian gravity limit. Moreover, there is a mass gap which separates the massless mode from the continuum. This is consistent with the fact that massive gravitons have not yet been found experimentally.

\section{Newtonian limit}

The gravitational potential of a source mass $M$ produced by the exchange of graviton reads as the Yukawa potential [12]:

$V(\rho)=\frac{M}{4 \pi} \sum_{m_{n}=0}^{+\infty} g^{2} \frac{e^{-m_{n} \rho}}{\rho}$

where $\rho$ is the distance on the brane from the source and sum is over the graviton spectrum found in the previous section and $g$ is coupling constant for the gravitational interaction and can be given as [10],

$g^{2}=\left.\frac{\kappa^{2}}{4} \tilde{D}_{\mu \nu}^{2}\right|_{y=0}= \begin{cases}\frac{\kappa^{2} k}{2} & \text { for } m^{2}=0, \\ \frac{\kappa^{2} k}{4}\left(\frac{m^{2}-2 k^{2}}{m^{2}-k^{2}}\right) & \text { for } m^{2}>2 k^{2},\end{cases}$

in which we have used (25)-(27).
The interaction term in the gravitational Lagrangian is [10]

$\mathscr{L}_{\text {int }}=\left.\frac{\kappa}{2} h_{\mu \nu}\right|_{y=0} T^{\mu \nu}$

where $T^{\mu \nu}$ is the energy-momentum tensor for matters on the 3-brane and $\kappa^{2}=\frac{8 \pi G}{k}$ such that

$S_{g}=\frac{1}{2 \kappa^{2}} \int \sqrt{\left|g^{(5)}\right|} R^{(5)} \mathrm{d} y \mathrm{~d}^{4} x$

because $R_{\mu \nu}^{(4)}$ is contained in $R_{\mu \nu}^{(5)}$ :

$$
\begin{aligned}
S_{g} & \sim \frac{1}{2 \kappa^{2}} \int e^{2 \sigma} \sqrt{\left|g^{(4)}\right|} e^{-\sigma} R^{(4)} \mathrm{d} y \mathrm{~d}^{4} x+\cdots \\
& =\frac{1}{2 \kappa^{2}} \int \sqrt{\left|g^{(4)}\right|} R^{(4)} \frac{1}{k} \mathrm{~d}^{4} x
\end{aligned}
$$

then

$\frac{1}{2 \kappa^{2} k}=\frac{1}{16 \pi G}$

where $G$ is the four-dimensional gravitational constant. Because of the partly continuous mass spectrum, the sum in (28) turns into an integration and it can be shown that the integral measure $d m$ is proportional to $k$ :

$$
\begin{aligned}
V(\rho) & =\frac{G M}{2 k}\left(\frac{2 k}{\rho}+\sum_{m_{n} \neq 0}^{+\infty} \frac{e^{-m_{n} \rho}}{\rho} \frac{g^{2}}{k}\right) \\
& =\frac{G M}{\rho}\left(1+\int_{\sqrt{2} k}^{+\infty} \frac{1}{2 k} \frac{m^{2}-2 k^{2}}{m^{2}-k^{2}} e^{-m \rho} \mathrm{d} m\right)
\end{aligned}
$$

the integration on the right hand side is accounted for by

$$
\begin{aligned}
\int_{\sqrt{2} k}^{+\infty} & \frac{1}{2 k} \frac{m^{2}-2 k^{2}}{m^{2}-k^{2}} e^{-m \rho} \mathrm{d} m=\frac{e^{-\sqrt{2} k \rho}}{2 k \rho} \\
& -\frac{1}{4} E i(1,(\sqrt{2}-1) k \rho) e^{-k \rho}+\frac{1}{4} E i(1,(\sqrt{2}+1) k \rho) e^{k \rho} \\
\simeq & e^{-2 \sqrt{2} k \rho}\left(-\frac{1}{4(\sqrt{2}-1) k \rho}+O\left(\left(\frac{1}{k \rho}\right)^{2}\right)\right) \text { for } k \rho \gg 1
\end{aligned}
$$

Then the potential for $\rho \gg 1 / k$ at first orders reads

$V(\rho) \simeq \frac{G M}{\rho}\left(1-\frac{e^{-2 \sqrt{2} k \rho}}{4(\sqrt{2}-1) k \rho}\right)$

So, as justified by Eq. (35), the MRS model meets the gravitational Newtonian limit, consistent with observations.

Experimentally bounds on $k$ can be found by a direct measurement of the gravitational force in torsion balance or cantilever experiments. These are based on the Yukawa potential 
as follows [13]:

$V(\rho)=-\frac{G M}{\rho}\left[1+\alpha e^{-r \lambda}\right]$

$\alpha$ being the strength and $\lambda$ the range of the additional potential. Their order of magnitudes were reported to be in different ranges, but typical orders can be considered as $\alpha \sim 10^{-2}$ and $\lambda \sim 50 \mu \mathrm{m}[14,15]$. Comparing with our model, we estimate $k$ to be a few $e V$. This looks very small and one may worry about KK modes production in accelerators. Why we have not yet seen them relies on their small coupling. As seen in Eq. (29), for fixed $k$ the effective gravitational coupling as a function of mass starts from 0 at $m^{2}=2 k^{2}$ to one half of the massless coupling for very large $\mathrm{KK}$ massive modes. Therefore, it is improbable to produce a $\mathrm{KK}$ graviton mode in the accelerators like LHC due to the weakness of the gravitational coupling.

\section{Discussion and conclusion}

In this paper, we constructed the gravitational perturbation of a MRS model, see (9), and found the equations of motion for different components of the metric perturbation in Eqs. (11)-(13). By the decomposition (14) and the unitary gauge, we solved the equations of motion. The mass spectrum for the graviton modes depicted a massless mode which dominates the Newtonian gravity limit, and a continuum that is responsible for a small correction to the Newtonian limit in short distances. Interestingly, there is a mass gap which separates the single massless mode from the mass continuum; this could be a reason why massive gravitons have not yet been detected experimentally. The higher dimensional corrections to the Newtonian gravity are exponentially suppressed due to the mass gap. A similar behavior has recently been reported in [16]. Roughly speaking this can be seen in any background with an effective potential which asymptotically grows to a positive constant. In our case, by considering Eq. (20) in $\sigma$ coordinates, it looks like a Schrödinger equation with a constant potential equal to $2 k^{2}$, which is exactly the gap found in the spectrum. In contrast, the massless graviton originates from the delta function localization of the brane.

Finally, it is worth mentioning that there is no negative mass-squared in the graviton spectrum, which indicates the stability of the model.

Open Access This article is distributed under the terms of the Creative Commons Attribution 4.0 International License (http://creativecomm ons.org/licenses/by/4.0/), which permits unrestricted use, distribution, and reproduction in any medium, provided you give appropriate credit to the original author(s) and the source, provide a link to the Creative Commons license, and indicate if changes were made.

Funded by SCOAP ${ }^{3}$.

\section{References}

1. N. Arkani-Hamed, S. Dimopoulos, G.R. Dvali, The Hierarchy problem and new dimensions at a millimeter. Phys. Lett. B 429 , 263 (1998). arXiv:hep-ph/9803315

2. L. Randall, R. Sundrum, A large mass hierarchy from a small extra dimension. Phys. Rev. Lett. 83, 3370 (1999). arXiv:hep-ph/9905221

3. M. Gogberashvili, Hierarchy problem in the shell universe model. Int. J. Mod. Phys. D 11, 1635 (2002). doi:10.1142/ S0218271802002992. arXiv:hep-ph/9812296

4. I. Oda, Gravitational localization of all local fields on the brane. Phys. Lett. B 571, 235 (2003). arXiv:hep-th/0307119

5. B. Bajc, G. Gabadadze, Localization of matter and cosmological constant on a brane in anti-de Sitter space. Phys. Lett. B 474, 282 (2000). arXiv:hep-th/9912232

6. M. Gogberashvili, D. Singleton, Brane in 6-D with increasing gravitational trapping potential. Phys. Rev. D 69, 026004 (2004). arXiv:hep-th/0305241

7. M. Gogberashvili, D. Singleton, Nonsingular increasing gravitational potential for the brane in 6-D. Phys. Lett. B 582, 95 (2004). doi:10.1016/j.physletb.2003.12.038. arXiv:hep-th/0310048

8. P. Jones, G. Munoz, D. Singleton, Triyanta, Field localization and Nambu Jona-Lasinio mass generation mechanism in an alternative 5-dimensional brane model. Phys. Rev. D 88, 025048 (2013). arXiv:1307.3599 [gr-qc]

9. M.N. Smolyakov, I.P. Volobuev, Is there the radion in the RS2 model? Cent. Eur. J. Phys. 2, 25 (2004). arXiv:hep-th/0302041

10. E.E. Boos, Y.A. Kubyshin, M.N. Smolyakov, I.P. Volobuev, Effective Lagrangians of the Randall-Sundrum model. Theor. Math. Phys. 131(2), 629-640 (2002)

11. S. Weinberg, Cosmology (Oxford University Press, Oxford, 2008)

12. L. Randall, R. Sundrum, An Alternative to compactification. Phys. Rev. Lett. 83, 4690 (1999). arXiv:hep-th/9906064

13. M. Azam, M. Sami, C.S. Unnikrishnan, T. Shiromizu, Proposal for an experiment to search for Randall-Sundrum-type corrections to Newton's law of gravitation. Phys. Rev. D 77, 101101 (2008). doi:10.1103/PhysRevD.77.101101. arXiv:0712.2118 [hep-th]

14. E.G. Adelberger, EOT-WASH Group Collaboration, Submillimeter tests of the gravitational inverse square law. doi:10. 1142/9789812778123_0002. arXiv:hep-ex/0202008

15. D.J. Kapner, T.S. Cook, E.G. Adelberger, J.H. Gundlach, B.R. Heckel, C.D. Hoyle, H.E. Swanson, Tests of the gravitational inverse-square law below the dark-energy length scale. Phys. Rev. Lett. 98, 021101 (2007). doi:10.1103/PhysRevLett.98.021101. arXiv:hep-ph/0611184

16. G. German, A. Herrera-Aguilar, D. Malagon-Morejon, R.R. MoraLuna, R. da Rocha, A de Sitter tachyon thick braneworld and gravity localization. JCAP 1302, 035 (2013). doi:10.1088/1475-7516/ 2013/02/035. arXiv:1210.0721 [hep-th] 